\title{
Lateral composition modulation in short period superlattices: The role of growth mode
}

\author{
C. Dorin and J. Mirecki Millunchick ${ }^{\mathrm{a})}$ \\ Department of Materials Science and Engineering, University of Michigan, Ann Arbor, Michigan 48109 \\ Y. Chen and B. G. Orr \\ The Harrison M. Randall Laboratory, University of Michigan, Ann Arbor, Michigan 48109 \\ C. A. Pearson \\ Department of Computer Science, Engineering Science and Physics, University of Michigan-Flint, Flint, \\ Michigan 48502
}

(Received 7 June 2001; accepted for publication 16 October 2001)

\begin{abstract}
The role of the growth mode on lateral composition modulation is studied in short period superlattices of AlAs/InAs and GaAs/InAs. Reflection high energy electron diffraction and scanning tunneling microscopy are used to monitor the growth mode and the quality of the interfaces. Cross-sectional transmission electron microscopy indicates that samples that grow via the layer-by-layer growth mode do not exhibit lateral composition modulation and the superlattice structure is well defined. Lateral composition modulation forms when roughening occurs during growth. However, too much roughening, i.e., three-dimensional island nucleation destroys the regularity of the composition modulation in both the lateral and vertical directions. These results are in general agreement with theoretical predictions. (C) 2001 American Institute of Physics.
\end{abstract}

[DOI: $10.1063 / 1.1425452]$

The growth of short period superlattices (SPSs) by molecular beam epitaxy frequently results in heterostructures that have spontaneously formed alloy composition variations in the plane of the film. Such lateral composition modulations are regular and robust for SPSs in which each layer thickness is on the order of a monolayer (ML) or two, and the layers have alternating compressive and tensile strain with respect to the substrate. Some examples include $\mathrm{AlAs} / \mathrm{InAs}^{1}$ and $\mathrm{GaAs} / \mathrm{InAs}^{2}$ multilayers on $\operatorname{InP}(001)$ substrates, and $\mathrm{GaP} / \mathrm{InP}$ on $\mathrm{GaAs}(001){ }^{3}$ Typical composition modulation wavelengths are 100-400 $\AA, 1,2$ and the modulation amplitude varies in composition from 0.4 to $0.6^{3,4}$ These types of structures have proven useful for optoelectronic devices with improved properties. ${ }^{5}$

It has been suggested that features at the growth front initiate lateral composition modulation, ${ }^{1}$ implying that surface instabilities play a major role in this phenomenon. ${ }^{6}$ In fact, several models predict that stress-driven morphological undulations are coupled to compositional modulations. ${ }^{7}$ For example, Spencer et al. found that compositional stress in alloy films further destabilizes morphological instabilities that arise due to misfit stresses. ${ }^{8}$ These models, however, consider the growth of single alloy layers and may not be valid for multilayer structures. Shilkrot, Srolovitz, and Tersoff ${ }^{9}$ applied a linear stability analysis to multilayer structures. They found that roughening of the surface depends greatly on the stress contributions from the buried interfaces. Under certain conditions, this model predicts that the multilayer structure exhibits lateral composition modulation as a result of variations in the thickness of the individual layer thicknesses as opposed to alloy decomposition.

${ }^{a)}$ Electronic mail: joannamm@umich.edu
Despite all of the recent work in this area, experimental verification of existing models has yet to emerge. In this letter, we examine the microstructure of AlAs/InAs and $\mathrm{GaAs} / \mathrm{InAs}$ SPS structures deposited on $\operatorname{InP}(001)$ substrates and show that the mode of growth, be it layer-by-layer or three-dimensional (3D) island formation, plays a significant role in the appearance of lateral composition modulation.

The films were grown using molecular beam epitaxy (MBE) on InP(001) substrates. Following the deposition of a lattice matched buffer layer, the SPS was grown by depositing $2 \mathrm{ML}$ of InAs followed by $2 \mathrm{ML}$ of AlAs or GaAs repeated 100 times. Several samples were grown at different temperatures of $500 \leqslant T \leqslant 520^{\circ} \mathrm{C}$. The structures were capped with $10 \mathrm{~nm}$ thick $\mathrm{In}_{0.53} \mathrm{Ga}_{0.47} \mathrm{As}$ in order to prevent oxidation of the Al-containing films. Reflection high energy electron diffraction (RHEED) was used to monitor the quality of the crystalline surface during growth. Cross-sectional transmission electron microscopy (XTEM) was performed on the samples in order to characterize the microstructure of the SPS. Samples were prepared using mechanical thinning, followed by Ar-ion milling at $4.5 \mathrm{kV}$ at angles of $3^{\circ}-5^{\circ}$. The character of the SPS interfaces was studied by in situ scanning tunneling microscopy (STM). The MBE growth of the samples to be characterized by STM proceeded as described above, except that only 30 periods of the SPS were deposited and the cap layer was omitted. After growth, the samples were transferred in ultrahigh vacuum to an adjoining STM chamber.

It was found that the growth mode is a significant factor in the final microstructure of compositionally modulated samples. For the AlAs/InAs SPS sample deposited at $T=500{ }^{\circ} \mathrm{C}$, the growth proceeded via the layer-by-layer growth mode. Figure 1(a) shows a plot of the intensity of the 


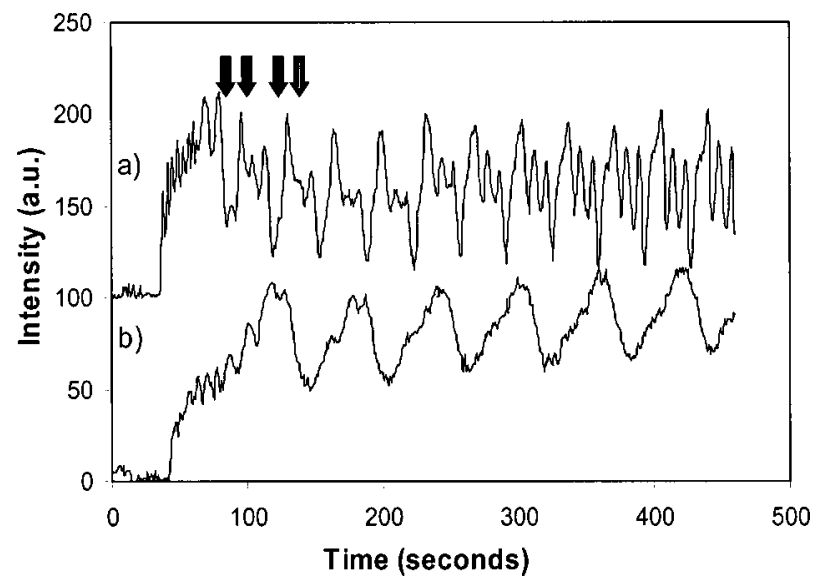

FIG. 1. Evolution of the RHEED specular spot as a function of time for an AlAs/InAs SPS grown at (a) $T=500{ }^{\circ} \mathrm{C}$ and (b) $520^{\circ} \mathrm{C}$.

specular reflection of the RHEED beam as a function of time. At the beginning of growth, RHEED oscillations arising from the initial buffer layer growth are apparent. Immediately following, the individual SPS layers were deposited, denoted by the arrows in Fig. 1(a). Four oscillations per period are observed, indicating that growth proceeds via the layer-by-layer mode. The surface reconstruction was $(2 \times 1)$ during the InAs deposition, and a weak $(3 \times 1)$ reconstruction during the AlAs deposition, and the final RHEED pattern was streaky, indicative of a smooth surface. The sample grown at higher temperature, $T=520^{\circ} \mathrm{C}$, did not grow via the layer-by-layer growth mode, as noted by the absence of growth oscillations in Fig. 1(b). Oscillations in the RHEED spectral intensity in Fig. 1(b) arise due to changes in the surface reconstruction from $(2 \times 1)$ reconstruction during the InAs growth and $(3 \times 1)$ during the AlAs growth. The final RHEED pattern was somewhat spotty, implying that some degree of roughening is present on the surface.

XTEM was performed in order to learn about the SPS microstructure for these films. Figure 2(a) is a bright field image of the (110) zone axis for the sample grown at $T=500{ }^{\circ} \mathrm{C}$. No visible defects or lateral inhomogeneities are observed in Fig. 2(a) and the individual SPS layers are clearly resolved at higher magnification (not shown). Furthermore, the SPS/cap interface is abrupt and planar. These results are in contrast to the lateral composition modulation observed in the samples grown at $T=520^{\circ} \mathrm{C}$. Figure $2(\mathrm{~b})$ is a dark field cross-sectional TEM image in the [1 10$]$ projection for the (002) reflection of this sample that shows strong regular and periodic lateral contrast along the [110] direction. The periodicity of the phase-separated region is $\approx 17 \mathrm{~nm}$, as calculated from $\mathrm{X}$-ray diffraction (XRD) reciprocal space maps (not shown). Lateral contrast due to composition modulation is also observed along the [1 $\overline{10}]$ direction (not shown), however, the periodicity is larger $(26 \mathrm{~nm})$. These results are in agreement with other reports of lateral composition modulation in the AlAs/InAs structure. ${ }^{10}$ For this sample, the SPS/cap interface is wavy, further indication of roughening in this structure.

Too much roughening or $3 \mathrm{D}$ island formation destroys the regularity of the lateral periodicity. This is demonstrated by the GaAs/InAs structure grown at $T=510{ }^{\circ} \mathrm{C}$, which also exhibits lateral composition modulation. The degree of
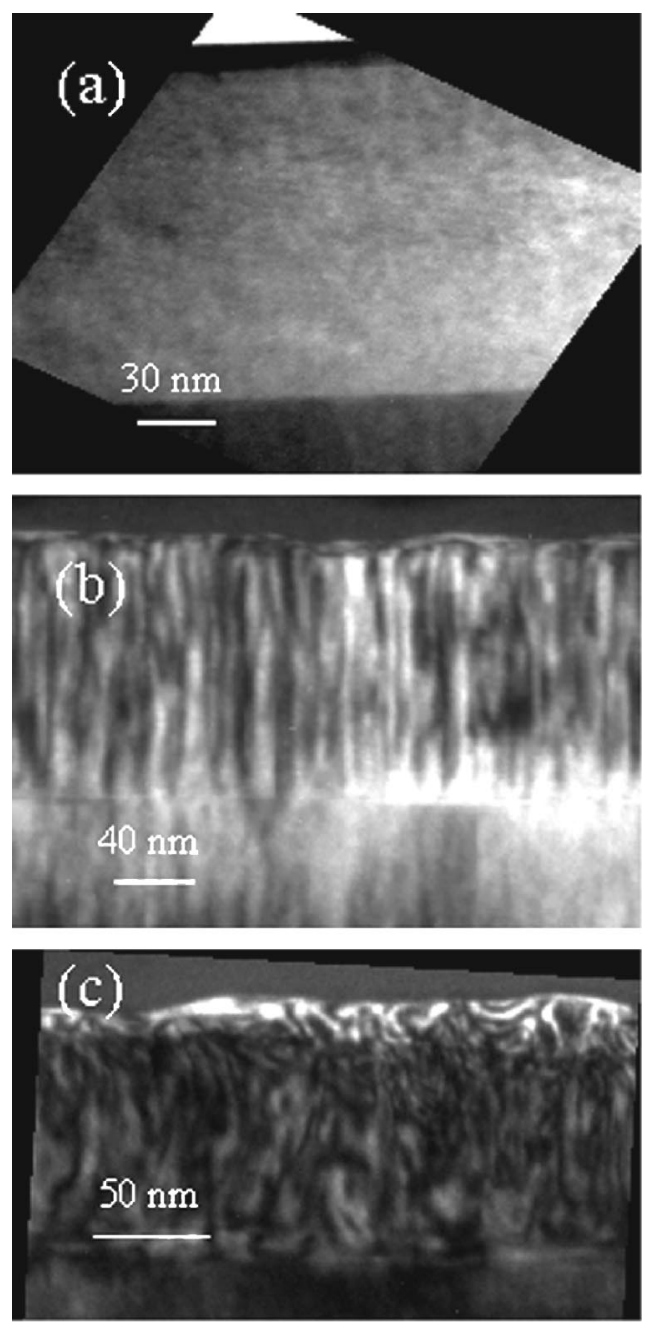

FIG. 2. Cross-sectional TEM images of an AlAs/InAs SPS structure: (a) bright field image in the [1] 0 ] projection for the sample grown at $T=500{ }^{\circ} \mathrm{C}$, (b) (002) dark field image in the [1 $\overline{10}$ ] projection for the sample grown at $T=520^{\circ} \mathrm{C}$, and (c) (002) dark field TEM image for a GaAs/InAs SPS grown at $T=510^{\circ} \mathrm{C}$.

roughening during growth was more pronounced compared to that of the Al-containing samples. In that sample, the RHEED pattern showed that the surface reconstruction was $(2 \times 1)$ during InAs deposition and $(3 \times 1)$ during GaAs deposition. However, the pattern became spotty, indicative of $3 \mathrm{D}$ island formation, after only a few periods. Figure 2(c) is a XTEM dark field image of this sample. Lateral composition modulation is present, as indicated by the variation in contrast. But the nature of the periodicity is considerably different than in the Al-containing samples. In this case, the lateral contrast is not correlated from the top to the bottom of the film and the lateral periodicity is erratic. This meandering of the phase-separated regions is most likely related to the $3 \mathrm{D}$ islands that are present on the surface.

The nature of the SPS interfaces was studied using in situ STM. Figures 3(a) and 3(b) are STM images of AlAs/ InAs SPSs deposited at different temperatures that illustrate differences in surface morphology. Figure 3(a) is from a sample grown at $T=500^{\circ} \mathrm{C}$. The presence of only a few terrace steps across the image, typical of the layer-by-layer growth mode, confirms that the surface is smooth. In stark contrast to this surface is the one shown in Fig. 3(b), which 

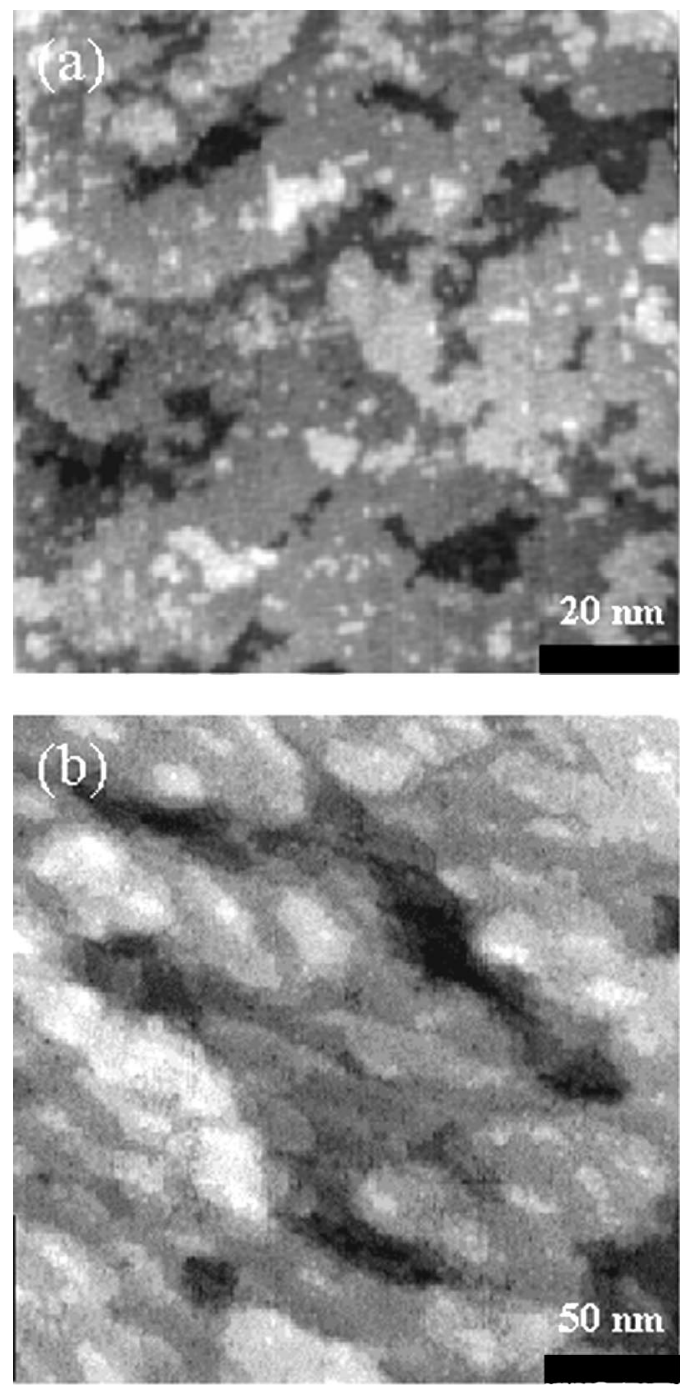

FIG. 3. In situ STM images of 30 period AlAs/InAs SPS structures grown at different temperatures. (a) $1000 \AA \times 1000 \AA$ image of the $T=500^{\circ} \mathrm{C}$ sample and (b) $2500 \AA \times 2500 \AA$ image of the $T=520^{\circ} \mathrm{C}$ sample. The surfaces were imaged at $-3.4 \mathrm{~V}$ sample bias and $100 \mathrm{pA}$ tunneling current.

is from a sample deposited at $T=520^{\circ} \mathrm{C}$. Many monolayerhigh islands are visible on the surface, and those islands are relatively uniform in size and regularly spaced. This spacing, approximately $25 \mathrm{~nm}$, is comparable to the periodicity of the lateral composition modulation determined from XRD reciprocal space maps and TEM images.

Lateral composition modulation has been postulated to arise due to the lattice strain between the individual SPS layers. $^{2}$ Therefore, it is remarkable that the microstructure of the AlAs/InAs and GaAs/InAs structures is so different considering that the lattice parameter (thus the strain) of AlAs and GaAs is nearly the same. These disparities are most likely due to the fact that $\mathrm{Al}$ and $\mathrm{Ga}$ have dissimilar surface diffusivities. ${ }^{11}$ Recent theoretical calculations suggest that the relative surface diffusivities play a large role in the development of composition modulation. Spencer et al. found that a difference in surface diffusivity between the alloy species can stabilize stress-driven morphological perturbations. ${ }^{8}$ Indeed, in this work the AlAs/InAs SPS structures, which have a larger difference between the activation energies of the migrating species, ${ }^{12}$ are smoother than the GaAs/InAs SPS structures.

Indium segregation may also be a significant factor in determining the final microstructure. ${ }^{13}$ It is known that In segregation is more pronounced in InAlAs alloys compared to in InGaAs alloys. ${ }^{12}$ Therefore, the individual SPS layers of the AlAs/InAs structures are expected to be more intermixed. This would lead to less lattice misfit between the layers and a larger critical thickness for roughening in the AlAs/InAs structures compared to in the GaAs/InAs structures.

These experimental observations are in qualitative agreement with the predictions of Shilkrot et al. ${ }^{9}$ In their model, there is a significant portion of phase space in which the multilayer stack remains planar despite the presence of stress between the individual layers, in agreement with Fig. 2(a). That model also predicts that for higher interlayer stress each layer develops surface undulations that result in lateral compositional variations, also in agreement with this work.

In conclusion, we show that roughening is necessary to induce lateral composition modulation. However, too much roughening destroys the vertical regularity and the lateral periodicity of the composition's modulation. The differences in the microstructure observed between the AlAs/InAs and GaAs/InAs SPS structures can be attributed to differences between the surface diffusivities of $\mathrm{Al}$ compared to those of $\mathrm{Ga}$ and to increased In surface segregation in Al-containing alloys. These observations are qualitatively consistent with theoretical models.

The authors gratefully acknowledge useful discussions with Corinna Wauchope, and the support of the National Science Foundation under Grant No. DMR9973352. One of the authors (C.A.P.) acknowledges support by a Faculty Development grant from the University of Michigan-Flint.

${ }^{1}$ J. Mirecki Millunchick, R. D. Twesten, D. M. Follstaedt, S. R. Lee, E. D. Jones, Y. Zhang, S. P. Ahrenkiel, and A. Mascarenhas, Appl. Phys. Lett. 70, 1402 (1997).

${ }^{2}$ K. Y. Cheng, K. C. Hsieh, and J. N. Baillargeon, Appl. Phys. Lett. 60, 2892 (1992).

${ }^{3}$ K. C. Hsieh, J. N. Baillargeon, and K. Y. Cheng, Appl. Phys. Lett. 57, 2244 (1990).

${ }^{4}$ R. D. Twesten, D. M. Follstaedt, S. R. Lee, E. D. Jones, J. L. Reno, J. Mirecki Millunchick, A. Norman, S. P. Ahrenkiel, and A. Mascarenahs, Phys. Rev. B 60, 13619 (1999).

${ }^{5}$ D. E. Wohlert, K. Y. Cheng, and S. T. Chou, Appl. Phys. Lett. 78, 1047 (2001)

${ }^{6}$ D. J. Srolovitz, Acta Metall. 37, 621 (1989); R. J. Asaro and W. A. Tiller, Metall. Trans. 3, 1789 (1972); M. A. Grinfeld, Sov. Phys. Dokl. 31, 831 (1986).

${ }^{7}$ J. E. Guyer and P. W. Voorhees, J. Cryst. Growth 187, 150 (1998); F. Glas, Phys. Rev. B 55, 11277 (1997); F. Leonard and R. C. Desai, ibid. 57, 4805 (1998).

${ }^{8}$ B. J. Spencer, P. W. Voorhees, and J. Tersoff, Phys. Rev. Lett. 84, 2449 (2000).

${ }^{9}$ L. E. Shilkrot, D. J. Srolovitz, and J. Tersoff, Phys. Rev. B 62, 8397 (2000).

${ }^{10}$ S. R. Lee, J. Mirecki Millunchick, R. D. Twesten, D. M. Follstaedt, J. L. Reno, S. P. Ahrenkiel, and A. G. Norman, J. Mater. Sci. 10, 191 (1999).

${ }^{11}$ T. Shitara, D. D. Vvendensky, J. H. Neave, and B. A. Joyce, Mater. Res. Soc. Symp. Proc. 312, 267 (1993).

${ }^{12}$ J.-M. Gerard, Appl. Phys. Lett. 61, 2096 (1992).

${ }^{13}$ C. Dorin and J. Millunchick, J. Appl. Phys. (in press). 